\title{
利用稳定同位素技术分析青海湖优势水鸟的营养级结构
}

\author{
杨月琴 ${ }^{1}$, 易现峰 ${ }^{1, *}$, 李 宁 $^{2}$ \\ （1. 河南科技大学 农学院, 河南 洛阳 471000；2. 青海大学 农牧学院, 青海 西宁 810007)
}

\begin{abstract}
摘要: 2008 年 4-6 月对青海湖湖区主要水鸟的稳定性碳和氮同位素组成进行了测定, 依据营养级间的同位 素富集效应以及营养级计算模型 $\left(\mathrm{TL}_{\text {水鸟 }}=3+\left(\delta^{15} \mathrm{~N}_{\text {水鸟 }}-1.78-\delta^{15} \mathrm{~N}_{\text {浮湔动物 }} / 3.8\right)\right.$, 分析并确定了主要水鸟在生态系统 中的营养级层次。相对于水生动物而言, 水鸟的 $\delta^{15} \mathrm{~N}$ 具有明显的富集效应, 而 $\delta^{13} \mathrm{C}$ 不显著。依据稳定性同位素 的营养富集效应模型，通过普通扸鹚（Phalacrocorax carbo）和青海湖裸鲤（Gymnocypris przewalskii）间 $\delta^{15} \mathrm{~N}$ 的 比对, 确定了该系统中排泄尿酸的水鸟稳定性氮同位素的富集因子为 $1.78 \%$ 。利用 $\delta^{15} \mathrm{~N}$ 建立了稳定同位素比值与 营养层次的关系。浮游植物、浮游动物、青海钩虾 (Gammarus suifunensis)、裸鲤和水鸟的营养层次分别为 1.64 - 2.06、2.35-2.83、2.63、3.64-4.20 和 3.35-4.93。由于湖区普通扸鸪、渔鸥 (Larus ichthyaetus)、棕头鸥 (Larus brunnicephalus) 和风头码嫄 (Podiceps cristatus) 的食物来源主要是湖体中的裸鲤, 由模型计算得到它们处在最 高的营养级阶层; 赤麻鸭（Tadorna ferruginea）、普通燕鸥（Sterna hirundo）和红脚璚（Tringa totanus）属杂食性 水鸟, 营养阶层居中; 而斑头雁 (Anser indicus) 由于主要以水生和陆生植物为食, 处在最低的营养阶层。裸鲤 资源未来的状况必将影响到普通扸鹚、渔鸥、棕头鸥, 以及风头辟䝠的生长、繁衍以及整个系统的群落结构和稳 定性。
\end{abstract}

关键词: 水鸟; 营养级; 裸鲤; 稳定性同位素; 青海湖

中图分类号: Q959.7; Q958.122; S124 文献标识码: A 文章编号: 0254-5853-(2009)04-0418-05

\section{Analysis on Trophic Structure of Main Waterbirds in Qinghai Lake Based on Stable Isotopic Technology}

\author{
YANG Yue-qin $^{1}$, YI Xian-feng ${ }^{1, *}$, LI Ning $^{2}$ \\ (1. College of Agriculture, Henan University of Science and Technology, Henan Luoyang 471000, China; \\ 2. Qinghai University, Xi'ning 810007, China)
}

\begin{abstract}
Stable carbon and nitrogen isotopes were measured to analyze trophic level of main waterbirds in Qinghai Lake in May-June, 2008, based on the model of isotopic enrichment and the fractionation factor. Our results indicated that $\delta^{15} \mathrm{~N}$ was significantly enriched in the muscle of waterbirds, but $\delta^{13} \mathrm{C}$ showed no obvious variation. We obtained a fractionation factor between Gymnocypris przewalskii and Phalacrocorax carbo, i.e., 1.78\%. Phytoplankton, zooplankton, Gammarus suifunensis, G. przewalskii and waterbirds were estimated at the trophic levels of 1.64-2.06, $2.35-2.83,2.63,3.64-4.20$ and 3.35-4.93 respectively. P. carbo, Larus ichthyaetus, Larus brunnicephalus and Podiceps cristatus occupied the highest trophic level due to their primary feeding on G. przewalskii. However, Tadorna ferruginea, Sterna hirundo, and Tringa totanus occupied the relatively high trophic levels. Anser indicus, mainly relying on plant materials as their food supply, was at the lowest trophic level. Our results predicted that the future resource situation of $G$. przewalskii would have great influence on the growth, reproduction and community structure of main waterbirds, especially for P. carbos, L. ichthyaetus, L. brunnicephalus and P. cristatus, consequently on the stability and diversity of the whole ecosystem.
\end{abstract}

Key words: Waterbird; Trophic level; G. przewalskii; Stable isotope; Qinghai Lake

稳定性碳、氮同位素技术被应用于生态系统生态学当中用来分析营养级关系, 是较为实用、准确

收稿日期: 2009-02-27; 接受日期: 2009-06-23

基金项目: 国家自然科学基金资助项目（30700078）; 河南省高校科技创新人才计划资助项目（2008 HASTIT003）

*通讯作者 (Corresponding author), E-mail: yxfeng1975@126.com

第一作者简介: 杨月琴 (1975-), 女, 硕士, 讲师, 主要从事稳定性同位素生态学研究 
和简单的一种方法 (Hobson, 1993; Johnson et al, 2004; Xu et al, 2005; Yi et al, 2006; Nonogaki et al, 2007; Paradis et al, 2008)。稳定性同位素技术是基于 生物体内天然存在的同位素比值与其食物密切相 关这一原理建立起来的。 $\delta^{13} \mathrm{C}$ 和 $\delta{ }^{15} \mathrm{~N}$ 在两营养级间 的变化常常分别以 $1 \% 0-2 \% 0$ 和 $3 \% 0-4 \%$ 存在。因 此, 顶级捕食物种 (top predator) 组织内 ${ }^{13} \mathrm{C}$ 和 ${ }^{15} \mathrm{~N}$ 的浓度是最高的。这样针对消费者组织中同位素的 测定就可以推断它们的食物信息以及在生态系统 中的营养级位置。

Wada et al (1987) 较早利用这一技术研究了南 极罗斯（Ross）海中生物群落的营养级关系。基于 稳定性同位素技术建立起的这种营养级不仅反映 了各物种在系统中的营养位置和取食关系, 而且也 可以反映出物种对物质的代谢水平以及环境因素 的差异所引起的营养状况和营养模式的改变

(Layman et al, 2007a)。因此, 由稳定性同位素富 集效应所构建的营养级是一种整合的营养级位置 关系。基于稳定性同位素推算出的营养级位置, 可 以相对容易地对生态系统的营养结构和营养生态 位进行描述 (McCutchan et al, 2003; Layman et al, 2007b)。

青海湖是我国最大的内陆咸水湖, 湖区栖息和 繁衍着成千上万的水鸟, 对维系湖区生态系统的稳 定和多样性起着至关重要的作用。湖区珍贵的鱼类 资源青海湖裸鲤 (以下简称裸鲤) 为这些水鸟提供 了丰富的食物来源。尽管国内外关于陆地和水体 (湖泊和海洋) 生态系统的稳定性同位素生态学有 了深入的研究 (Cai et al, 2001; Hobson et al, 2001; Xu et al, 2005; Yi \& Yang, 2006; Yi et al, 2006), 但作 为我国最大的内陆咸水湖, 青海湖生态系统的稳定 性同位素生态学研究目前还是空白。传统的手段和 方法 (如胃容物分析和直接观察等) 可准确地解决 短时间内的动物食性组成, 但对于长时间序列, 尤 其是生活史阶段的食性信息的推断存在缺陷。稳定 性同位素技术可以为食性分析提供新的研究手段 和更为真实的记录。本文尝试用稳定性碳、氮同位 素的比率来建立青海湖生态系统水鸟的营养级关 系, 为分析水鸟食性信息以及评价湖区生态系统安 全积累基础资料并奠定一定理论基础。

\section{1 材料与方法}

\section{1 研究区域概况}

青海湖位于青海省东北部的大通山、日月山和 青海南山之间 (东经 $99^{\circ} 36^{\prime}-100^{\circ} 47^{\prime}$ 、北纬 $36^{\circ} 32^{\prime}-37^{\circ} 15^{\prime}$ )。湖水面积 $4392 \mathrm{~km}^{2}$, 平均 海拔 $3260 \mathrm{~m}$, 属高原内陆深水型湖泊, 环湖地区地 势表现为西北高而东南低, 四周群山环绕, 形成了 封闭式的山间内陆盆地。湖区有布哈河、黑马河和 铁卜恰河等大小河流 30 余条, 西北面鸟岛、鸬鹚 岛以及湖心的海心山和三块石等, 这些都是野生水 鸟重要的繁殖地。湖区全年日照时数 $3000 \mathrm{~h}$ 以上, 年日照百分率达 $68 \%$ - $69 \%$, 年均温在 $1.1{ }^{\circ} \mathrm{C}$ 左右, 东部和南部气温稍高。

\section{2 稳定同位素测定}

于 2008 年 4-6 月, 经相关部门批准, 获取青 海湖湖区 8 种主要优势种水鸟和部分裸鲤样品, 同 时选取湖区主要浮游动、植物样品作为分析材料。 鸟类以胸肌作样品, 裸鲤以胴体白肌肉作样品, 浮 游动物、无脊椎动物及昆虫则取全体。所有样品经 恒温干燥箱 $60-80^{\circ} \mathrm{C}$ 烘干至恒重, 充分研磨, 过 100 目篮备用。以 MAT253 同位素质谱仪和元素分 析仪进行测定。由于样品与标准参照物之间比率差 异较小, 所以稳定同位素丰度表示为样品与标准之 间偏差的千分数:

$$
\delta \mathrm{R}_{\text {sample }}(\% \mathrm{o})=\left(\mathrm{R}_{\text {sample }}-\mathrm{R}_{\text {standard }}\right) / \mathrm{R}_{\text {standard }} \times 1000
$$
其中 $\delta R_{\text {sample }}$ 为被测材料的同位素组成; $R_{\text {sample }}$ 为被 测样品的同位素比值; $R_{\text {standard }}$ 为标准品的同位素比 值。

$$
\begin{aligned}
& \delta^{13} \mathrm{C}_{\text {样品 }}=\left\{\left({ }^{13} \mathrm{C} /{ }^{12} \mathrm{C}_{\text {样品 }}\right) /\left({ }^{13} \mathrm{C} /{ }^{12} \mathrm{C}_{\text {标准 }}\right)-1\right\} \times 1000 \\
& \delta^{15} \mathrm{~N} \text { 样品 }=\left\{\left({ }^{15} \mathrm{~N} /{ }^{14} \mathrm{~N} \text { 样品 }\right) /\left({ }^{15} \mathrm{~N} /{ }^{14} \mathrm{~N} \text { 标准 }\right)-1\right\} \times 1000
\end{aligned}
$$

\section{3 营养级划分}

生物体在食物网中的营养层次可以用稳定氮 同位素比值的高低进行表征。营养级分析方法参考 以下计算公式:

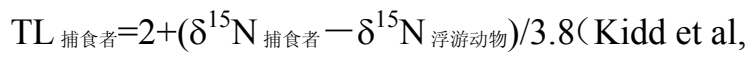
1995; Fisk et al, 2001)

式中, 2 假定为浮游动物营养层次; 3.8 为 $\delta^{15} \mathrm{~N}$ 富集因子。由于水鸟与水生生物不同, 属于恒温动 物并且排泄的是尿酸而不是尿素, 所以它与被捕食 鱼类的 $\delta^{15} \mathrm{~N}$ 富集值和水生食物网的富集因子不同 (Hobson, 1992)。本文以普通鸹兹和裸鲤的 $\delta^{15} \mathrm{~N}$ 之 间的差值测得水鸟捕食鱼类的 $\delta^{15} \mathrm{~N}$ 富集因子为 1.78\%。该富集因子比 Mizutani et al（1991）经过 23 年观测得出 $\delta^{15} \mathrm{~N}$ 富集因子 $2.4 \%$ 要小。因此, 可 以得到水鸟捕食生物的营养层次关系公式: 
$\mathrm{TL}_{\text {水岛 }}=\mathrm{TL}_{\text {水中捕食者 }}+1 ; \delta^{15} \mathrm{~N}_{\text {水鸟 }}=\delta^{15} \mathrm{~N}_{\text {水中捕食者 }}+1.78$ 因此, 水鸟的 $\delta^{15} \mathrm{~N}$ 与营养层次关系为:

$\mathrm{TL}_{\text {水鸟 }}=3+\left(\delta^{15} \mathrm{~N}_{\text {水鸟 }}-1.78-\delta^{15} \mathrm{~N}_{\text {浮游动物 }}\right) / 3.8$

\section{2 结 果}

\section{1 水鸟的稳定性同位素组成}

结果表明 (表 1 ), 青海湖湖区主要水鸟 $\delta^{13} \mathrm{C}$ 和 $\delta^{15} \mathrm{~N}$ 的数值在种间变化较大: 其中, 斑头雁的 $\delta^{13} \mathrm{C}$ 值明显高于其他几种水鸟, 前者为 $-16.30 \%$, 后者 的平均值为 $-23.50 \%$ 。然而, 斑头雁的 $\delta^{15} \mathrm{~N}$ 值在 8 种水鸟中最低, 为 $8.46 \%$; 赤麻鸭、红脚捅和普通 燕鸥的 $\delta^{15} \mathrm{~N}$ 值居中, 分别为 $9.85 \% 0 、 11.00 \% 0$ 和 $10.00 \%$; 明显低于其他几种水鸟的平均值 (13.95\%o)。普通扸兹的 $\delta^{13} \mathrm{C}$ 和 $\delta^{15} \mathrm{~N}$ 值分别为 $-23.19 \%$ 和 $14.45 \%$ 。棕头鸥及其幼鸟、风头辟崕、 渔鸥及其幼鸟的 ${ }^{13} \mathrm{C}$ 和 $\delta^{15} \mathrm{~N}$ 值比普通鸬鹚略低, 分 别为 $-22.45 \%$ 和 $13.25 \%$ 及其 $-22.72 \%$ 和 $13.42 \%$ 、 $-22.87 \%$ o 和 $14.16 \%$ 、 $-23.97 \%$ 和 $14.27 \%$ 及 其 $-23.01 \%$ 和 $14.14 \%$ 。作为湖区重要的鱼类资源和水 鸟的食物来源, 青海湖裸鲤的 $\delta^{13} \mathrm{C}$ 值介于 $-24.16 \%$ 和-18.66\% 之间， $\delta^{15} \mathrm{~N}$ 值介于 $11.56 \%$ 和 $13.67 \%$ 之 间, 且裸鲤稳定性碳、氮同位素比值具有随体重增 加而升高的趋势。无脊椎动物青海钩虾的 $\delta^{13} \mathrm{C}$ 和 $\delta^{15} \mathrm{~N}$ 值分别为 $-20.67 \%$ 和 $7.75 \%$ 。浮游动物的稳定 性同位素组成为 $-20.02 \%$ 和 $5.33 \%$; 浮游植物的稳
定性同位素组成为 $-18.23 \%$ 和 $5.56 \%$ 。

\section{2 水鸟营养级阶层}

结果表明, 青海湖区的普通鸬鹚所处的营养级 位置最高, 为 4.93 (表 1), 说明它的主要食物来源 依赖于湖体中的裸鲤。斑头雁、赤麻鸭、红脚柕岛和 普通燕鸥的营养级远低于棕头鸥、渔鸥、凤头辟鹤 和渔鸥幼鸟所处的营养级位置。其中斑头雁所处的 营养级最低, 反映出其主要以来植物性食物为食的 取食策略。棕头鸥、渔鸥和风头辟塬鸟所处的营养级 分别为 4.62、4.88 和 4.85, 说明它们也处在较高的 营养阶层。但它们的营养级位置比普通鸬鹚要低一 些。因此, 它们的食物来源除了大部分裸鲤以外, 还应包括湖体内部的无脊椎浮游动物和其他水生 昆虫等（图 1)。基于稳定性碳、氮同位素在二维坐 标上的分布情况, 我们可以把青海湖 8 种主要鸟类 划分成 3 个明显的营养级群体 (图 2)。普通鸬鹚、 棕头鸥、渔鸥和风头辟塬处在最高的营养级群体, 赤麻鸭、红脚捅和普通燕鸥处在第二营养级群体, 而斑头雁处在最低的营养级群体, 进而反映了几种 主要水鸟生态位之间的分离和重叠程度。

\section{3 讨 论}

尽管一些海洋生态系统或单一食物关系的研 究表明 $\delta^{13} \mathrm{C}$ 值随营养层次的富集因子约为 $1 \%$

表 1 青海湖生态系 8 种主要水鸟的稳定性碳氮同位素组成及其营养级水平

Tab. 1 Stable carbon and nitrogen isotopic patterns and trophic levels of eight main waterbirds in Qinghai Lake ecosystem

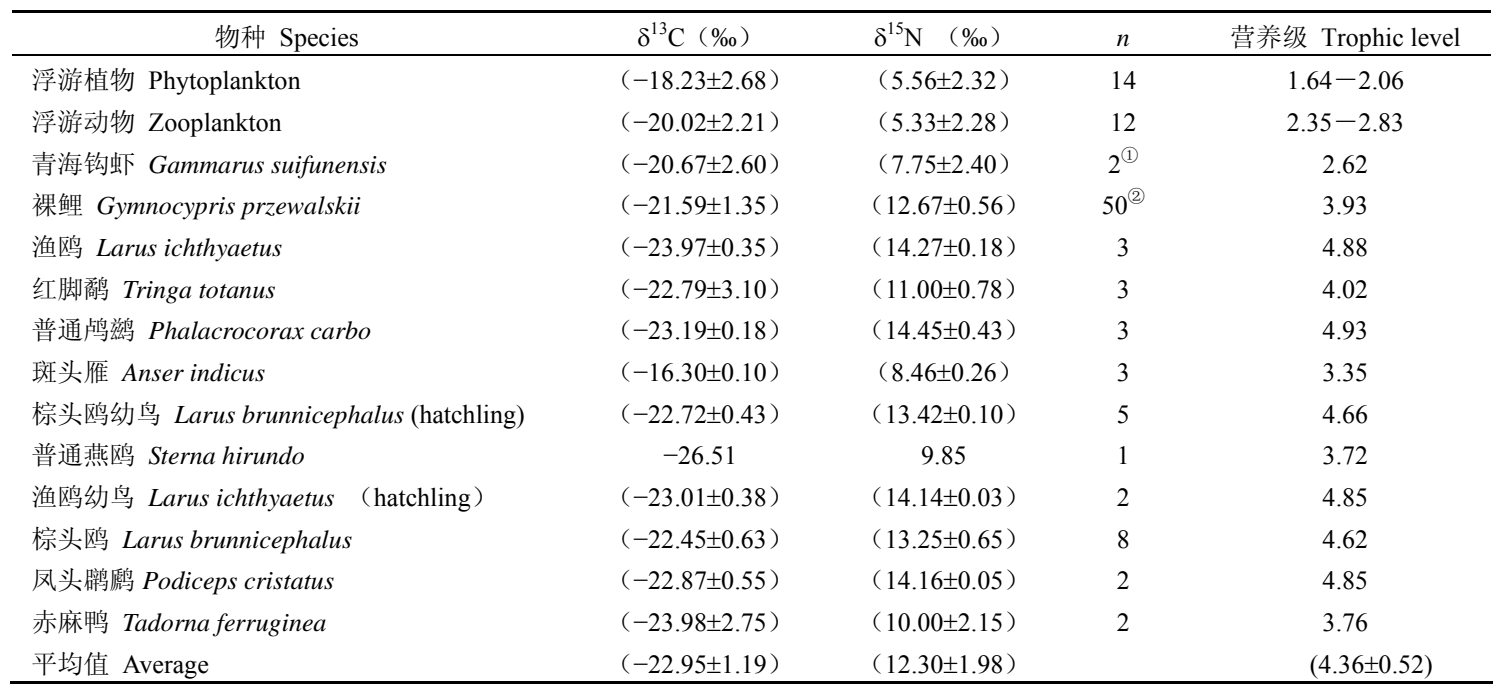

(1)为 12 个个体的混合物（two mixed sample of 12 individuals); (2)体重介于 $0.04-1.6 \mathrm{~kg}$ 之间 (body weight from $0.04-1.6 \mathrm{~kg}$ )。 


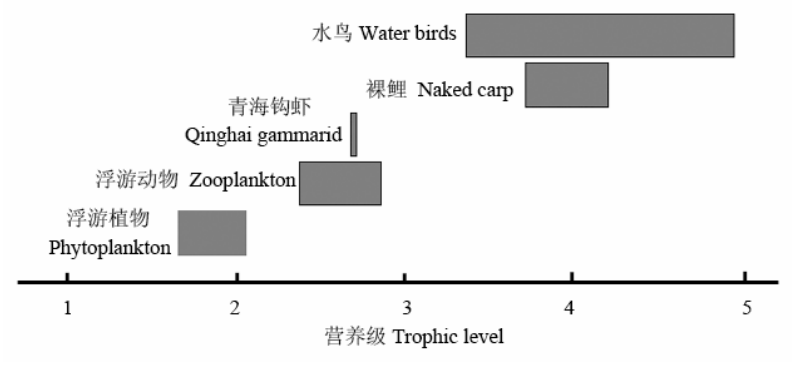

图 1 青海湖水鸟的营养级与其他生物的关系

Fig. 1 Trophic relationship between waterbirds and other organisms in Qinghai Lake

(Deniro \& Epstein, 1978; Rau et al, 1983; Mills et al, 1984 ), 但我们的结果并没有发现 $\delta^{13} \mathrm{C}$ 值稳定的富 集现象, 植食性的斑头雁具有异常高的 $\delta^{13} \mathrm{C}$ 值。这 与 Hobson \& Welch (1992) 在北冰洋以及 Wada et al (1987) 在南冰洋海洋生态系统的发现一致。与他 人的研究结果 (Miyake \& Wada, 1967; Wan et al, 2005 ) 不同的是, 浮游动物的稳定性碳同位素比值 明显低于浮游植物, 这可能与高原湖体温度低, 肌 肉内脂肪含量高引起的 $\delta^{13} \mathrm{C}$ 值降低有关 (Wada et al, 1987 ), 也可能与取食湖底沉积有机物有关。结果 表明, 青海湖鱼类的 $\delta^{13} \mathrm{C}$ 值明显要比渤海湾的低

(Wan et al, 2005), 进一步支持我们的推论。与碳 同位素相比, $\delta^{15} \mathrm{~N}$ 值在水鸟与裸鲤之间富集效应较 弱, 最大的富集值为 $1.78 \%$, 低于 Mizutani et al （1991）报道的 2.4\%o，呈现一种特殊的稳定性氮 同位素富集效应。但水鸟与无脊椎的钩虾以及浮游 动物之间存在明显的富集效应（图 1), 在 6\%o-7\% 之间, 因此在它们之间必然还存在 $1-2$ 个营养阶 层 (裸鲤)。成鸟和幼鸟之间的稳定性碳和氮同位 素值差异不明显, 说明两者之间的同位素代谢水平 相当。

我们的研究结果表明, 青海湖生态系统的营养 级可分为 5 级。 8 种主要水鸟的营养级阶层出现分 离, 反映出它们对裸鲤以及其他水生生物依赖性的 不同。据 Li et al（1998）报道, 斑头雁主要以禾本 科和莎草科植物的叶、茎, 青草和豆科植物种子等 植物性食物为食, 有时也吃贝类、软体动物和其他

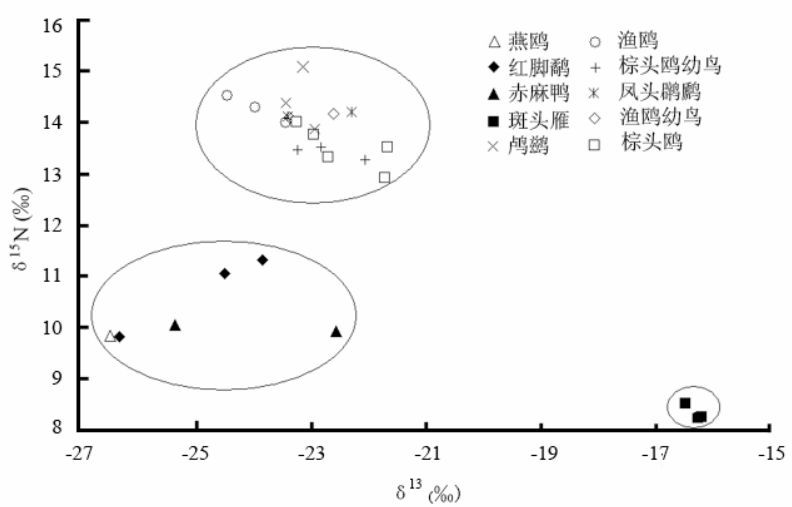

图 2 青海湖水鸟的 $\delta^{13} \mathrm{C}$ 和 $\delta^{15} \mathrm{~N}$ 的二维分布

Fig. 2 Two dimensional distribution of $\delta^{13} \mathrm{C}$ and $\delta^{15} \mathrm{~N}$ of eight waterbirds in Qinghai Lake ecosystem

小型无脊椎动物。但本研究中同位素组成表明, 其 食物来源主要应为浮游植物和大型沉水植物, 因为 斑头雁的碳同位素比值与浮游植物非常接近, 而与 陆源植物的差异较远 (Yi et al, 2003a)。普通鸬兹主 要通过潜水捕食大型鱼类, 因而其具有较高的稳定 性同位素比值。渔鸥以鱼类为主要食物, 发现鱼群 立即俯冲水中捕捉或叨水面上的伤病鱼及死鱼, 从 不潜水捕捉，因此其同位素比值也较高。棕头鸥的 捕食能力远不及普通鸹兹和鱼鸥, 主食鱼及水生软 体动物。所以它们经常追随扸兹、鱼鸥之后寻找剩 余食物, 同位素比值较为接近。Li (2003) 在梁子 湖的调查发现, 凤头辟鹛的食物主要为草食纤维, 而非动物性食物。本研究的同位素结果表明, 凤头 辟嫄的 $\delta^{13} \mathrm{C}$ 值为 $(-22.87 \pm 0.55) \%$ ，远高于青海 湖流域陆生植物和昆虫 $\delta^{13} \mathrm{C}$ 的平均值 $(-26.51 \%$ 和 $-25.23 \%$ ) (Yi et al, 2003a, b; Yi et al, 2006), 说明 凤头辟鹤的主要食物不是陆生草食纤维或昆虫, 而 主要依赖于湖体中的鱼、虾和部分水生植物为食。 这种不一致性可能与研究地点的植被状况以及湖 区生物组成不同有关。本研究结果表明, 普通燕鸥 的稳定性碳同位素与我们先前在该地区测定的陆 源植物非常接近 (Yi et al, 2003a, b), 而与水生生物 相去较远 (表 1), 说明其食物来源可能主要依赖于 陆源的 $\mathrm{C}_{3}$ 植物或昆虫, 水生生物的贡献相对较少。 由此可见, 处于第 4 阶层的青海湖裸鲤在为湖区鸟 类提供了主要的食物来源的同时, 对维持水鸟种群 多样性以及系统的稳定性方面起着重要的作用。 


\section{参考文献:}

Cai DL, Wang R, Bi HS. 2001. Trophic relationships in the Bohai ecosystem: preliminary investigation from $\delta^{13} \mathrm{C}$ and $\delta^{15} \mathrm{~N}$ analysis [J]. Acta Ecol Sin, 21: 1354-1359.

Deniro MJ, Epstein S. 1978. Influence of diet on the distribution of carbon isotopes in animals [J]. Geochim Cosmochim Acta, 42: 495-506.

Fisk AT, Hobson KA, Norstrom RJ. 2001. Influence of chemical and biological factors on trophic transfer of persistent organic pollutants in the northwater Polynya marine food web [J]. Environ Sci Tech, 35: 732-738.

Hirons AC, Schell DM, Finney BP. 2001. Temporal records of $\delta^{13} \mathrm{C}$ and $\delta^{15} \mathrm{~N}$ in North Pacific pinnipeds: inferences regarding environmental change and diet [J]. Oecologia, 129: 591-601.

Hobson KA, Welch HE. 1992. Determination of trophic relationships within a high arctic marine food web using $\delta^{13} \mathrm{C}$ and $\delta^{15} \mathrm{~N}$ analysis [J]. Mar Ecol Progr Ser, 84: 9-18.

Hobson KA. 1993. Trophic relationships among high arctic seabirds: insights form tissure-dependent stable-isotope models [J]. Mar Ecol Progr Ser, 95: 7-18.

Johnson MW, Hesslein RH, Dick TA. 2004. Host length, age, diet, parasites and stable isotopes as predictors of yellow perch (Perca flavescens Mitchill), trophic status in nutrient poor Canadian Shield lakes [J]. Environ Biol Fish, 71: 379-388.

Kidd KA, Schindler DW, Hesslein RH, Muir DCG. 1995. Correlation between stable nitrogen isotope ratios and concentrations of organochlorines in biota from a freshwater food web [J]. Sci Total Environ, 160/161: 381-390.

Layman CA, Arrington AA, Montan CG, Post DM. 2007a. Can stable isotope ratios provide for community-wide measures of trophic structure [J]? Ecology, 88: 42-48.

Layman CA, Quattrochi, JP, Peyer CM, Allgeier JE. 2007b. Niche width collapse in a resilient top predator following ecosystem fragmentation [J]. Ecol Lett, 10: 937-944.

Li FS, Nie H, Ye CH. 1998. Feeding habit of Bar-headed Geese wintering at Caohai Guizhou province [J]. Chn J Zool, 33: 29-33. [李风山, 聂 卉, 叶长虹. 1998. 贵州草海斑头雁的冬季食性分析. 动物学杂志, 33: 29-33.]

Li ZW. 2003. Re-understanding reproductive distribution and food structure of Podiceps cristatus [J]. Wildlife, 3: 27-29. [李振文. 2003. 重新认识 凤头辟源的繁殖分布和食物结构. 野生动物, 3: 27-29.]

McCutchan JJH, Lewis JWM, Kendall C, McGrath CC. 2003. Variation in trophic shift for stable isotope ratios of carbon, nitrogen, and sulfur [J]. Oikos, 102: 378-390.

Mills EL, Pittman K, Tan FC. 1984. Food-web structure on the Scotian
Shelf, eastern Canada: A study using ${ }^{13} \mathrm{C}$ as a food-chain tracer $[\mathrm{J}]$. Rapp P-v Réun Cons Int Explor Mer, 183: 111-118.

Miyake Y, Wada E. 1967. The abundance ratio of $15 \mathrm{~N} / 14 \mathrm{~N}$ in marine environments [J]. Rec Oceanogr Works Japan, 9: 32-59.

Mizutani H, Kabaya Y, Wada E. 1991. Nitrogen and carbon isotope compositions relate linearly in cormorant tissues and its diet [J]. Isotopenpraxie, 4: 166-168.

Nonogaki H, Nelson JA, Patterson WP. 2007. Dietary histories of herbivorous loricariid catfishes: evidence from $\delta^{13} \mathrm{C}$ values of otoliths [J]. Environ Biol Fish, 78: 13-21.

Paradis Y, Bertolo A, Magnan P. 2008. What do the empty stomachs of northern pike (Esox lucius) reveal? Insights from carbon $\left(\delta^{13} \mathrm{C}\right)$ and nitrogen $\left(\delta^{15} \mathrm{~N}\right)$ stable isotopes [J]. Environ Biol Fish, 83: 441-448.

Rau GH, Mearns AJ, Young DR, Olson RJ, Schafer HA, Kaplan IR. 1983. Animal ${ }^{13} \mathrm{C} /{ }^{12} \mathrm{C}$ correlates with trophic level in pelagic food webs $[\mathrm{J}]$. Ecology, 64: 1314-1318.

Wada E, Terazaki M, Kabaya Y, Nemoto T. $1987 .{ }^{15} \mathrm{~N}$ and ${ }^{13} \mathrm{C}$ abundances in the Antarctic Ocean with emphasis on the biogeochemical structure of the food web [J]. Deep Sea Res, 34: 829-841.

Wan Y, Hu JY, An LH, An W, Yang M, et al. 2005. Trophic level of main organisms in the Bohai ecosystem using stable carbon and nitrogen isotopes [J]. Chn Sci Bull, 7: 708-712. [万 祎, 胡建英, 安立会, 安 伟, 杨 敏, 伊藤光明, 服部达也, 陶 澍. 2005. 利用稳定氮和碳 同位素分析渤海湾食物网主要生物种的营养层次. 科学通报, 7: 708-712.]

Xu J, Xie P, Zhang M, Yang, H. 2005. Variation in stable isotope signatures of seston and a zooplanktivorous fish in a eutrophic Chinese lake [J]. Hydrobiologia, 541: 215-220.

Yi XF, Yang YQ, Zhang XA. 2006. Modeling trophic positions of the alpine meadow ecosystem combining stable carbon and nitrogen isotope ratios [J]. Ecol Model, 193: 801-808.

Yi XF, Yang YQ. 2006. Enrichment of stable carbon and nitrogen isotopes of plant populations and plateau pikas along altitudes [J]. J Ani Feed Sci, 15: 661-667.

Yi XF, Yang YQ, Zhang XA, Li LX, Zhao L. 2003a. No $C_{4}$ plants found at the Haibei alpine meadow ecosystem research station in Qinghai, China: evidence from stable carbon isotope studies [J]. Acta Bot Sin, 45: 1291-1296.

Yi XF, Zhang XA, Li LX, Li MC, Zhao L. 2003b. Analysis on food web structure in alpine meadow ecosystem: evidence from stable carbon isotope signatures [J]. Zool Res, 25: 1-6. [易现峰, 张晓爱, 李来兴, 李明财, 赵 亮. 2003. 高寒草甸生态系统食物链结构分析一来自 稳定性碳同位素的证据. 动物学研究, 25: 1-6.] 